Conclusions Undoubtedly some of the results of this audit may have been impacted by the COVID 19 pandemic, which commenced in March 2019 and naturally had a significant impact on service delivery and provision during the months that followed.

With regards to this audit, this is likely to be particularly relevant to the children's blood pressure and heart rate measurements and follow up appointments. To overcome this, Saturday Blood Pressure clinics were established locally and measurements were recorded along with centiles. Any children and young people who were due/overdue these checks were invited to attend to ensure ongoing holistic care. Escalation process is implemented to alert the child's paediatrician in case of concerns and for timely action.

\section{ELEVATED BP AMONG CHILDREN AND ADOLESCENTS WITH ADHD DURING THE INITIAL UK COVID-19 LOCKDOWN: SOCIOECONOMIC EFFECTS AND POSSIBLE INDICATOR OF MENTAL STRESS}

${ }^{1}$ Michael Ogundele, ${ }^{2}$ Hani F Ayyash, ${ }^{3}$ Samuele Cortese. ${ }^{1}$ Bridgewater Community Healthcare NHS Foundation Trust; ${ }^{2}$ Integrated Department of Paediatrics, Mid and South Essex University Hospitals Group, Southend University Hospital NHS Foundation Trust, Westcliff-On-Sea, Essex, UK; ${ }^{3}$ Child and Adolescent Psychiatry, University of Southampton and Solent NHS Trust, UK

\subsection{6/archdischild-2021-rcpch.864}

Background Several non-infectious adverse outcomes have been reported among children and young people (CYP) during the COVID-19 pandemic, including mental health symptoms, even though they are less prone to acute infections. The COVID-19 pandemic lockdown (CPL) was inevitably introduced globally to reduce the risk of infection transmission and necessitated significant restrictions on all social contacts, including school and workplace closures. Chronic stress including social isolation has been hypothesised to constitute a risk factor for hypertension (HT). Therefore, we hypothesised that children and adolescents with ADHD might display higher rates of HT following the CPL.

Objectives We aimed to assess the impact of COVID-19 pandemic lockdown (CPL) on the cardiovascular changes among CYP managed for ADHD within a Community Child Unit.

Methods We carried out a prospective cardiovascular assessment of a cohort of 41 (88\% male) CYP attending routine medical reviews for ADHD treatment in community paediatric clinic in the North-West England within the first 6 weeks of the first wave of the UK-wide Covid-19 lockdown in MarchMay 2020.

Their blood pressures (BP) were measured with regularly calibrated electronic sphygmomanometers based on standard clinical procedures and compared to $\mathrm{BP}$ recorded within the previous one year. Definition of Hypertension (HT) or PreHT was based on the British reference charts for CYP. The CYP were followed up with non-clinic-based BP monitoring at home or by GP. ${ }^{1}$

We assessed the socio-economic status (SES) of each CYP, using the last version of the Index of Multiple Deprivation (IMD) 2019 ranking order of their residential Lower-Layer Super Output Areas, based on the postcodes. Spearman's rank correlation coefficient was used to determine the relationship between the different proportions in a sample in discrete nominal groups. Statistical significance was accepted at the $\mathrm{p}$ value of $<0.05$.

Results Mean age of children studies was 12 years (age range; 5 years 9 months to 18 years), 37/out of 41 (90\%) were on ADHD medications, $92.5 \%$ were on various psycho-stimulant formulations while $7.5 \%$ were on non-stimulants. All the medications were within the lower range of normally approved doses.

There was a statistically significant increase in the HR, diastolic and systolic BP of all the CYP with ADHD during the CPL. There was two- to four-fold increase in the number of ADHD CYP with elevated BP measured after the UK CPL compared to the measurements in the previous 12 months (table 1). This increase in measured BP and HR could not be attributed to any prescribed ADHD medications as the patients had been on the same medications for an average of more than 16 months, all within the lower range of normal doses. There was statistically significant correlation between the duration of the CPL in weeks and the pre-/post-CPL differences between both the systolic and diastolic BP (table 1).

\begin{tabular}{|c|c|c|c|c|c|c|c|}
\hline Wks_Post CPL ${ }^{\$}$ & No-Pts & Avg-HR & $\Delta \mathrm{HR}$ & Avg-SB & Avg- $\Delta S B$ & Avg-DiB & Avg- $\Delta \mathrm{Di}$ \\
\hline 1 & 12 & 84.4 & 1.6 & 116.0 & 15.4 & 73.4 & 14.4 \\
\hline 2 & 11 & 80.6 & 1.9 & 108.5 & 15.2 & 71.5 & 12.9 \\
\hline 3 & 8 & 92.6 & 9 & 110.0 & 5.3 & 75.8 & 11.3 \\
\hline 4 & 9 & 87.7 & 8.2 & 114.2 & 8.7 & 70.3 & 12.1 \\
\hline 5 & 1 & 57 & 9 & 117.0 & -15 & 69 & -9 \\
\hline TOTAL & 41 & 84.8 & 4.8 & 112.7 & 11.2 & 72.5 & 12.3 \\
\hline Rho & & -0.2 & 0.82 & 0.4 & -0.9 & -0.7 & -0.9 \\
\hline$P$ value & & 0.75 & 0.09 & 0.5 & $0.04^{* *}$ & 0.19 & $0.04^{* *}$ \\
\hline
\end{tabular}

\section{Legend:}

Avg - Average (excluding missing readings); No - Number; Pts - ADHD patients; Rho Spearman Coefficient of correlation; CPL - Covid-19 Pandemic lockdown; HR - Heart Rate; SB - Systolic BP; DiB - Diastolic BP

\$ Week 1 starting from 26th March 2020; ** Statistically significant; $\Delta$ Difference between pre- and post-CPL measurements.

73 percent (30/41) of the CYP lived in the most deprived Quintile of the community while 7\% (3/41) lived in the most affluent Quintile. There was no statistically significant difference between the different areas of socio-economic deprivation and the observed changes in the CVS status of the CYP.

Conclusions The pandemic lockdown was associated with statistically significant increase in the $\mathrm{HR}$, diastolic and systolic $\mathrm{BP}$ of all the CYP with ADHD. This finding suggests that there was progressively increasing stress experienced during the lockdown by CYP with ADHD.

It highlights the need of adequate provisions for effective preventive measures to address the significant stress among CYP during future lockdowns or similar emergencies.

This study did not confirm any significant association between different areas of socio-economic deprivation and the observed changes in the CVS status of the CYP.

\section{REFERENCE}

1. Mahmood S et al. Non-pharmacological management of hypertension: in the light of current research. Ir J Med Sci 2019 May;188(2):437-452. 